Pesq. Vet. Bras. 29(9):731-735, setembro 2009

\title{
Alterações morfológicas de tecido laminar do casco e parâmetros clínicos e laboratoriais de equinos com síndrome cólica letal ${ }^{1}$
}

\author{
Luciane M. Laskoski2*, Carlos A. Araújo Valadão ${ }^{3}$, Rosemeri O. Vasconcelos ${ }^{4}$, \\ Rodrigo N. Pereira ${ }^{5}$, Andrea D.P. Uribe Dias $^{5}$ e Antônio C. Alessi ${ }^{4}$
}

\begin{abstract}
Laskoski L.M., Valadão C.A.A., Vasconcelos R.O., Pereira R.N., Dias A.D.P.U. \& Alessi A.C. 2009. [Morphologic alterations of the hoof lamellar tissue, and clinic and laboratorial analyses of horses with lethal colic syndrome.] Alterações morfológicas de tecido laminar do casco e parâmetros clínicos e laboratoriais de equinos com síndrome cólica letal. Pesquisa Veterinária Brasileira 29(9):731-735. Departamento de Clínica e Cirurgia Veterinária, Faculdade de Ciências Agrárias e Veterinárias, Universidade Estadual Paulista, Via de Acesso Prof. Paulo Donato Castellane s/n, Jaboticabal, SP 14884-900, Brazil. E-mail: lucianelaskoski@ hotmail.com

The gastrointestinal diseases of horses are aggravated by complications such as laminitis. The laminitis etiopathogeny are connected with lamellar basement membrane degradation by matrix metalloproteinases (MMPs). Inflammatory cytokines and leukocytes enzymes can active MMPs. The object of this study was to evaluate morphological changes on lamellar tissue of horses with colic syndrome and its association with clinical and laboratorial parameters. It was observed intensive destruction of lamellar architecture, mainly on animals with severe physical and laboratorial alterations, such as delayed capillary refill time, congested mucous membrane, tachycardia, hemoconcentration and low count of platelet and leukocytes. The results sign to the most likely moment of development of lamellar tissue injuries in horses with colic syndrome, which can be adopted preventive measures against laminitis.
\end{abstract}

INDEX TERMS: Colic syndrome, endotoxemia, horse, laminitis, laminar histopathology.

RESUMO.- As afecções gastrintestinais dos cavalos são agravadas por complicações como a laminite, cuja etiopatogenia está relacionada à degradação da membrana basal do tecido laminar por metaloproteinases (MMPs). A ativação das MMPs pode ocorrer devido à liberação local de citocinas inflamatórias ou enzimas provenientes de leucócitos infiltrados no tecido laminar. O objetivo deste trabalho foi avaliar as alterações morfológicas do teci-

\footnotetext{
${ }^{1}$ Recebido em 10 de dezembro de 2008.

Aceito para publicação em 18 de maio de 2009.

${ }^{2}$ Departamento de Clínica e Cirurgia Veterinária, Faculdade de Ciências Agrárias e Veterinárias (FCAV), Universidade Estadual Paulista (Unesp), Via de Acesso Prof. Paulo Donato Castellane s/n, Jaboticabal, SP 14884900, Brasil. *Autor para correspondência: lucianelaskoski@ hotmail.com

${ }^{3}$ Departamento de Clínica e Cirurgia, FCAV, Unesp-Jaboticabal, SP.

${ }^{4}$ Departamento de Patologia Veterinária, FCAV, Unesp-Jaboticabal, SP.

${ }^{5}$ Departamento de Clínica e Cirurgia Veterinária, FCAV, UnespJaboticabal, SP.
}

do laminar de equinos com síndrome cólica letal e sua provável associação com parâmetros clínicos e laboratoriais. Observou-se intensa destruição da arquitetura laminar, principalmente nos animais com alterações físicas e laboratoriais mais acentuadas, como tempo de preenchimento capilar prolongado (TPC), membranas mucosas congestas, taquicardia, hemoconcentração e redução nas contagens de plaquetas e leucócitos. Os resultados sinalizam o provável momento do desenvolvimento de lesões do tecido laminar em equinos com síndrome cólica, no qual é possível adotar medidas preventivas contra a laminite.

TERMOS DE INDEXAÇÃO: Síndrome cólica, endotoxemia, equinos, laminite, histopatologia laminar.

\section{INTRODUÇÃO}

Cerca de $25 \%$ dos animais acometidos por enfermidades gastrintestinais desenvolvem laminite, com variação des- 
ta porcentagem de acordo com a causa da afecção gastrintestinal. Na duodenojejunite, $30 \%$ dos animais são afetados (Fernandes et al. 2003); e na compactação do cólon maior 5\% (Rhoads et al. 1997). No entanto, são escassas as citações sobre as alterações histológicas do tecido laminar associadas a afecções de ocorrência natural, apenas em indução experimental de lesão intestinal (Rio Tinto et al. 2004).

A etiologia da laminite é desconhecida, todavia, existem diversas teorias a seu respeito. Uma dessas, a teoria enzimática, sugere a ativação de MMPs por leucócitos, citocinas inflamatórias ou toxinas bacterianas, causando degradação do colágeno presente na membrana basal e perda das interdigitações entre as lâminas dérmicas e epidérmicas (Pollitt 1999), e sugere-se que estes fatores ativadores das MMPs possam estar no tecido laminar em menos de 24 horas após o início do processo primário (Loftus et al. 2007a, Nourian et al. 2007).

A endotoxemia parece estar associada ao desenvolvimento da laminite aguda, cujo aparecimento ocorre em algumas doenças tais como pneumonias e afecções intestinais (Parsons et al. 2007). As alterações de parâmetros laboratoriais são descritas na ativação inflamatória sistêmica presente na endotoxemia e estão relacionadas ao agravamento do quadro clínico, como trombocitopenia e leucocitose ou leucopenia. A trombocitopenia relaciona-se com o aparecimento de tromboembolia e com a diátese hemorrágica presente na sepse (Dong et al. 2005, Koury et al. 2006, Campebell et al. 2007), sendo identificada também em animais durante a indução de laminite (Moore et al. 1981). A leucopenia, causada pela migração e ativação de leucócitos nos tecidos, pode levar a liberação de metaloproteinases que causam lesão tecidual (Zerpa et al. 2005).

Este trabalho tem por objetivo identificar possíveis alterações morfológicas do tecido laminar de equinos com síndrome cólica letal, avaliando a intensidade das lesões e associando-as aos parâmetros de exames físicos e laboratoriais de cada animal.

\section{MATERIAL E MÉTODOS}

Para este estudo foram utilizados seis equinos atendidos no Hospital Veterinário "Governador Laudo Natel", da Faculdade de Ciências Agrárias e Veterinárias (FCAV/UNESP), Campus de Jaboticabal, SP. Os equinos apresentavam idades de 1-13 anos, sendo dois da raça Mangalarga Paulista e quatro da raça Quarto de Milha, e todos morreram por afecções gastrintestinais.

Os valores dos exames físicos e laboratoriais deste estudo foram obtidos durante o período de atendimento dos equinos, sendo avaliadas a frequência cardíaca, a frequência respiratória, a coloração de membranas mucosas, a motilidade intestinal, e o tempo de preenchimento capilar (TPC); determinados o hematócrito, a proteína total; e realizada a contagem de plaquetas e de leucócitos. Os resultados hematológicos foram comparados com aqueles descritos por Jain (1986).

A extração do tecido laminar para o estudo histológico foi realizada em todos os cascos dos membros torácicos e pélvicos de cada equino. Os cascos foram seccionados no plano medial à semelhança da técnica de Pollitt (1996). Os fragmentos de tecido laminar foram fixados em solução de formol tamponado a $10 \%$ por 24 horas e processados para inclusão em parafina. As amostras foram cortadas a $5 \mathrm{~mm}$ de espessura e coradas pela hematoxilina e eosina (HE), para visualização das alterações morfológicas do tecido laminar. A técnica de coloração por ácido periódico de Schiff (PAS) foi utilizada para a análise da integridade da membrana basal. Os escores de lesão foram baseados na classificação de Pollitt (1996), com modificações relativas a escores intermediários, para classificar as lesões com maior acurácia (Quadro 1).

As alterações morfológicas, com respectiva intensidade, foram associadas às alterações dos exames físicos e laboratoriais de cada animal.

\section{RESULTADOS}

Todos os parâmetros físicos dos equinos apresentaram alterações, com exceção da frequência respiratória do Equino 1 que estava dentro dos valores normais (16mpm) (Quadro 2). Na frequência cardíaca observaram-se variações entre 60 e 124 bpm. Quanto à avaliação de membrana mucosa, a coloração observada com frequência foi

Quadro 1. Escores das lesões histológicas observadas no tecido laminar de equinos com cólica (adaptado de Pollitt 1996)

\begin{tabular}{|c|c|}
\hline Escore & Características histológicas \\
\hline 0 & $\begin{array}{l}\text { LES }^{b} \text { com extremidade arredondada, LEPC retilínia e com extremidade arredondada, núcleos das } \text { CB }^{d} \text { ovalados } \\
\text { e apicais, } \text { MB }^{\mathrm{e}} \text { com contorno bem definido. }\end{array}$ \\
\hline $0,5^{\mathrm{a}}$ & $\begin{array}{l}\text { Presença de núcleos de CB arredondados, mas com localização ainda apical em alguns segmentos, e de algumas } \\
\text { LES com extremidade levemente afilada. }\end{array}$ \\
\hline 1,0 & $\begin{array}{l}\text { LES afiladas com redução do espaço pertencente a LDS }{ }^{\dagger} \text {, núcleos de CB arredondados e próximos à MB, LEP com } \\
\text { extremidade levemente ondulada. }\end{array}$ \\
\hline $1,5^{\mathrm{a}}$ & $\begin{array}{l}\text { LES marcadamente afiladas e alongadas, com redução da LDS entre as LES, núcleos de CB arre-dondados e pró- } \\
\text { ximos à MB, LEP com extremidade afilada e ondulada, entretanto, a MB se encontra com distância normal da LEP } \\
\text { (espaço compreendido por } 2 \text { CB). MB contorno irregular e borrado. }\end{array}$ \\
\hline 2,0 & $\begin{array}{l}\text { Todas as alterações anteriores, com aumento significativo da distância entre o eixo queratinizado das LEP e MB, e } \\
\text { esta se encontra serrilhada e ausente em vários segmentos na coloração de PASg. }\end{array}$ \\
\hline 3,0 & $\begin{array}{l}\text { Intensa destruição da arquitetura laminar, ausência marcante de MB, extremidade das LES sensivelmente afiladas } \\
\text { e alongadas, formando uma massa amorfa de tecido entre LEP e LDP, causado também pelo descolamento das } \\
\text { LES do eixo da LEP }\end{array}$ \\
\hline
\end{tabular}

Pesq. Vet. Bras. 29(9):731-735, setembro 2009 
Quadro 2. Idade, diagnóstico e tempo de evolução da afecção gastrintestinal e resultados de exames físicos e laboratoriais dos equinos com síndrome cólica

\begin{tabular}{|c|c|c|c|c|c|c|}
\hline \multirow{2}{*}{$\begin{array}{l}\text { Equino/ldade } \\
\text { Diagnóstico }\end{array}$} & 01 (5 anos) & \multirow{2}{*}{$\begin{array}{c}02 \text { (7 anos) } \\
\text { Rompimento } \\
\text { de ceco }\end{array}$} & \multirow{2}{*}{$\begin{array}{c}03 \text { (13 anos) } \\
\text { Torção da } \\
\text { raiz mesentérica }\end{array}$} & \multirow{2}{*}{$\frac{04 \text { (10 anos) }}{\text { Duodenojejunite }}$} & \multirow{2}{*}{$\begin{array}{c}05 \text { (8 anos) } \\
\text { Rompimento } \\
\text { de cólon maior }\end{array}$} & \multirow{2}{*}{$\begin{array}{c}06 \text { (1 ano) } \\
\begin{array}{c}\text { Peritonite } \\
\text { difusa }\end{array}\end{array}$} \\
\hline & $\begin{array}{l}\text { Torção da } \\
\text { aiz mesentérica }\end{array}$ & & & & & \\
\hline Evolução (dias) & 7 & 2 & 7 & 2 & 2 & 2 \\
\hline $\mathrm{FC}^{\mathrm{a}}(30-40 \mathrm{bpm})$ & 76 & 60 & 100 & 65 & 80 & 124 \\
\hline $\mathrm{FR}^{\mathrm{b}}(10-20 \mathrm{mpm})$ & 16 & 28 & 70 & 30 & 60 & 36 \\
\hline Muc. $^{c}$ (rósea) & Cong. ${ }^{j}$ & Cong. & Cong. & Cong. & Cong. & Cian. ${ }^{k}$ \\
\hline Mot. Int. ${ }^{d}$ & Aus. & Aus. & Aus. & Aus. & Aus. & Dim. ${ }^{m}$ \\
\hline $\operatorname{TPC}^{\mathrm{e}}(1-2 \mathrm{~s})$ & 3 & 4 & 3 & 4 & 4 & 4 \\
\hline $\mathrm{Ht}^{\dagger}(30$ a $45 \%)$ & 40,9 & 63,3 & 47,3 & 47,1 & 58,6 & 32,7 \\
\hline $\mathrm{Pt}^{\mathrm{g}}(5,8-8,7 \mathrm{~g} / \mathrm{dL})$ & 5,0 & 7,2 & 8,2 & 6,6 & 7,8 & 9,3 \\
\hline Plaq $^{h}\left(100-350 \times 10^{3} / \mathrm{il}\right.$ & 129 & 233 & 183 & 86 & 226 & 304 \\
\hline Leuc $^{i}\left(5,4-14,3 \times 10^{3} / \mathrm{il}\right)$ & 3,5 & 2,4 & 14,1 & 1,1 & 2,7 & 9,2 \\
\hline
\end{tabular}

a Frequência cardíaca, em bpm (batimento por minuto); ${ }^{b}$ frequência respiratória, em mpm (movimentos por minuto); ${ }^{c}$ coloração de membrana mucosa; ${ }^{d}$ motilidade intestinal; ${ }^{e}$ tempo de preenchimento capilar, em segundos; ${ }^{f}$ hematócrito; 9 proteína total; ${ }^{\mathrm{h}}$ contagem de plaquetas; ${ }^{\mathrm{i}}$ contagem de leucócitos; ${ }^{\mathrm{j}}$ congesta; ${ }^{\mathrm{k}}$ cianóticas; ' ausente; ${ }^{\mathrm{m}}$ diminuída. Valores entre parênteses representam os parâmetros normais para eqüinos adultos.

vermelho-escura (congestão), e o TPC variou de 3 a 4 segundos. A motilidade intestinal estava ausente na maioria dos animais. Apenas o Equino 4 mostrou sinais de laminite, com troca de apoio dos cascos e aumento perceptível do pulso digital nos membros pélvicos. Não houve variação na temperatura corpórea destes equinos. Todos os animais apresentaram refluxo, por meio de sondagem naso-gástrica, com odor alterado e coloração escura, acastanhada, com volume médio de 8 litros.

Nos exames laboratoriais verificou-se hemoconcentração ou tendência a essa alteração na maioria dos animais (aumento do $\mathrm{Ht}$ e Pt), bem como redução da contagem de plaquetas, ou valores próximos do nível inferior, e
Quadro 3. Escore histológico de acordo com o membro dos equinos com síndrome cólica

\begin{tabular}{ccccccc}
\hline Equino & 01 & 02 & 03 & 04 & 05 & 06 \\
\hline TD $^{\mathrm{a}}$ & 1,5 & 3,0 & 1,5 & 1,5 & 1,5 & 0,5 \\
TE $^{\mathrm{b}}$ & 1,0 & 3,0 & 1,5 & 1,5 & 3,0 & 0,5 \\
PD $^{\mathrm{c}}$ & 0,5 & 3,0 & 0,5 & 3,0 & 0,5 & 0,5 \\
PE $^{\mathrm{d}}$ & 1,0 & 3,0 & 1,0 & 3,0 & 0,5 & 0,5
\end{tabular}

${ }^{\text {a }}$ Torácico direito, ${ }^{b}$ torácico esquerdo, ${ }^{c}$ pélvico direito, ${ }^{d}$ pélvico esquerdo.

de leucócitos. A única alteração laboratorial observada no animal 6 foi o aumento da proteína total (Quadro 2).

As alterações do tecido laminar receberam escores entre 0,5 e 3 (Quadro 3). Nas amostras de tecido laminar

Fig.1. Fotomicrografia do tecido laminar de equinos com síndrome cólica. (A) Escore 0,5; nota-se a presença de núcleos de células basais (CB, setas) ovalados (setas contínuas pretas/insert) e lâminas epidérmicas secundárias (LES) de extremidade arredondada (seta pontilhada preta), mas também a presença de núcleos de CB esféricos (setas contínuas brancas/ insert) e afilamento de algumas LES (seta pontilhada branca). HE, obj.40x. (B) Escore 0,5, apresentando membrana basal (MB, setas) de contorno definido (insert). PAS, obj.40x. (C) Escore 3; nota-se intensa destruição da arquitetura laminar, com LES descoladas (seta) da lâmina epidérmica primária formando massa amorfa (insert), com a presença de congestão venosa (seta). HE, obj.40x. (D) Escore 3 , com ausência de MB (seta) em alguns segmentos e contorno acentuadamente irregular (insert). PAS, obj.40x.
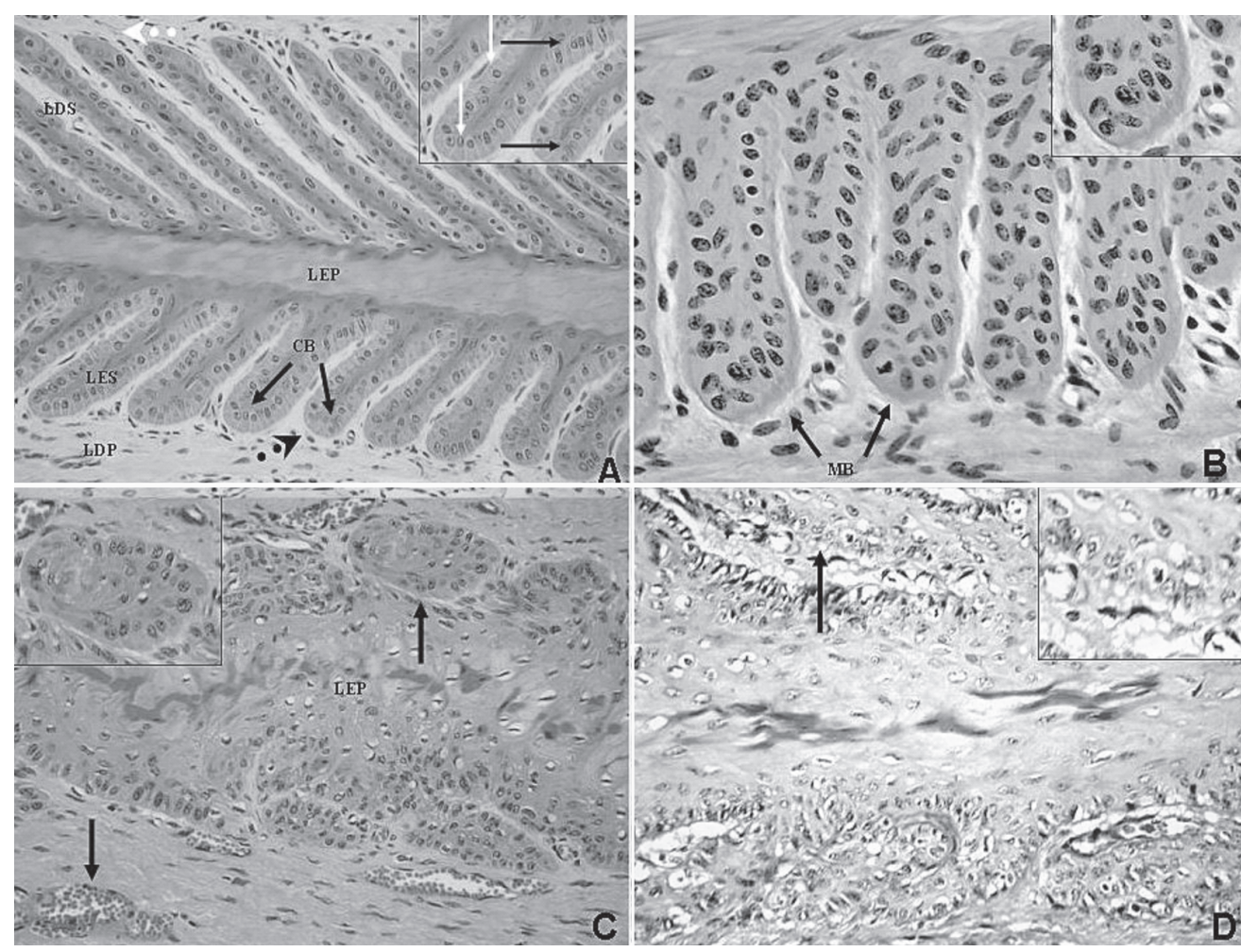
com escore 3 verificou-se intensa destruição da arquitetura laminar e perda das interdigitações de lâminas epidérmicas secundárias (LES) e das lâminas dérmicas secundárias (LDS) (Fig.1C). A membrana basal (MB), responsável pela conexão entre as LES e LDS estava ausente em muitos segmentos (Fig.1D). As lesões de menor intensidade caracterizaram-se pela presença de núcleos das células basais esféricos (CB) e não ovalados (Fig.1A) como no tecido normal, LES afiladas, ondulação da lâmina epidérmica primária (LEP) e contorno irregular e serrilhado da MB (Fig.1D). A congestão vascular foi observada com intensidade acentuada no escore 3 e intensidade moderada nos escores 1 e 1,5. Não foram registrados coágulos, necrose e edema em nenhuma das camadas do tecido laminar.

As lesões laminares classificadas com escores superiores a 1 , especialmente o escore 3 , foram observadas em animais que obtiveram alterações dos exames físicos e laboratoriais, como taquicardia, congestão de membranas mucosas, TPC elevado, hemoconcentração e redução na contagem de plaquetas e de leucócitos.

\section{DISCUSSÃO}

As alterações físicas observadas nos eqüinos deste estudo tais como o aumento das frequências cardíaca e respiratória, TPC elevado, congestão de mucosas, ausência de motilidade intestinal e presença de refluxo naso-gástrico permitem sugerir envolvimento de ativação inflamatória sistêmica por provável endotoxemia (Moore 2001), a qual apresenta ocorrência frequente em afecções gastrintestinais de equinos e está associada com o desenvolvimento de laminite (Parsons et al. 2007). Bailey et al. (2009) em estudo com laminite induzida pela administração de oligofrutose, observaram traços de endotoxina em torno de oito horas após a indução da doença. O aumento do TPC pode indicar deficiência na perfusão periférica, hipovolemia e deterioração do sistema cardiovascular (Moore et al. 1994).

A desidratação é comumente observada em cavalos com abdômen agudo, sendo indicada pelo aumento do hematócrito e, ocasionalmente, da proteína total que nem sempre estará elevada em decorrência da perda protéica encontrada em lesões gastrintestinais (Alves et al. 2005). A contagem de plaquetas foi inferior ao valor normal em um animal e próxima ao valor inferior em outros dois equinos, achado que se relaciona ao agravamento do quadro clínico e morte (Koury et al. 2006, Silva et al. 2007). Por esse fato sugere-se que os animais poderiam desenvolver tromboembolia e diátese hemorrágica, como nos quadros terminais de endotoxemia (Dong et al. 2005). A trombocitopenia também foi observada em equinos com laminite induzida por sobrecarga de carboidratos (Moore et al. 1981).

Leucopenia foi observada na maioria dos animais, sendo comum a todos com escore 3 , e também em um equino com escore 1,5 (Equino 1). Esta alteração pode estar associada à ativação inflamatória sistêmica causada pela endotoxemia, com sequestro tecidual de leucócitos (Zerpa et al. 2005, Campebell et al. 2007). Fernandes et al. (2003) associaram a leucopenia com a severidade do quadro endotoxêmico em cavalos com duodenojejunite.

O Equino 6 apresentou alterações laboratoriais mínimas, com aumento de proteína total. Igualmente não se observou alteração considerável do tecido laminar (escore 0,5$)$. O diagnóstico foi peritonite, cuja causa primária não foi determinada. Desta forma, provavelmente, a reduzida ativação inflamatória neste animal, sugerida apenas pela hiperproteinemia, possa ter sido responsável pelas ínfimas alterações do tecido laminar.

Com exceção de um equino, os outros apresentaram alterações microscópicas no tecido laminar de escore igual ou superior a 1, o que, segundo Pollitt (1996) caracteriza a condição clínica de laminite. Os animais com rompimento intestinal e duodenojejunite apresentaram escore 3 em um ou mais cascos, provavelmente em razão do maior dano intestinal gerado por estas enfermidades.

A ausência de edema no tecido laminar corrobora com os achados de Pollitt (1996), em que os espaços aumentados observados entre as lâminas não estão associados ao conteúdo líquido. A congestão venosa, acentuada no escore 3 , pode estar associada à vasodilatação, que facilita o transporte de substâncias, como toxinas, citocinas e leucócitos ao tecido laminar, ativando as metaloproteinases que degradam o colágeno presente na MB (Pollitt 1996). Hipotensão arterial foi observada em animais com laminite induzida por sobrecarga de carboidratos, antecedendo os sinais clínicos, com aumento da temperatura da borda coronária dos cascos (Garner et al. 1975). Nos quadros endotoxêmicos graves ocorre incapacidade de manutenção do tônus simpático, levando a vasodilatação periférica (Maitra et al. 2000). Assim, sugere-se que a congestão venosa observada seja oriunda do colapso circulatório devido ao quadro endotoxêmico.

Não foi possível identificar, pela coloração de hematoxilina e eosina, a presença significativa de leucócitos no tecido laminar. Alguns trabalhos demonstram que existem técnicas mais precisas para demonstrar a presença do infiltrado inflamatório no tecido laminar (Black et al. 2006, Loftus et al. 2006), as quais não foram realizadas neste estudo. No entanto, a presença da leucopenia aqui observada sugere infiltração tecidual leucocitária (Zerpa et al. 2005). Segundo Hurley et al (2006), a presença de leucócitos no interstício laminar é coincidente com a leucopenia na laminite induzida por extrato de nogueira preta. Com o mesmo modelo, Galey et al. (1991), constataram redução de leucócitos duas horas antes do aparecimento dos sinais clínicos da enfermidade podal. Desta forma, semelhante à falência orgânica observada na sepse humana, a injúria laminar pode ser causada pela liberação de numerosos mediadores inflamatórios pelos leucócitos, como espécies reativas de oxigênio e metaloproteinases (Belknap \& Black 2005, Zerpa et al. 2005, Loftus et al. 2006). Um achado importante que contribui para a ocorrência de lesões graves no tecido laminar de equinos é a ausência da enzima superóxido dismutase (SOD) nesse 
tecido, a qual promove proteção contra os efeitos deletérios das espécies reativas de oxigênio, agindo como antioxidante (Loftus et al. 2007b). Desse modo, pode-se explicar a possível razão pela qual a sequela mais frequente das afecções com envolvimento sistêmico no equino seja a laminite (Belknap \& Black 2005).

Uma vez que a maioria dos animais do estudo apresentava comprometimento sistêmico grave, evidenciado pelas alterações de exames físicos e laboratoriais, o desenvolvimento de alterações histopatológicas foi acentuado e, provavelmente, suficiente para deflagrar a laminite. No entanto, não houve a manifestação de sinais clínicos de laminite em todos os animais, mascarados provavelmente pela gravidade do quadro clínico. Supõe-se que se os animais houvessem sobrevivido provavelmente os sinais se manifestariam, especialmente as alterações morfológicas laminares classificadas como escore 3.

\section{CONCLUSÕES}

A intensidade das lesões observadas no tecido laminar de equinos com afecções gastrintestinais parece estar associada ao grau de comprometimento sistêmico, especialmente com a evolução do quadro para choque séptico.

Os achados laboratoriais, em especial a leucopenia, podem ser indicadores úteis do provável desenvolvimento concomitante de laminite.

A associação destes achados fornece indícios preliminares que sinalizam a adoção de cuidados adicionais ao paciente equino, visando prevenir a laminite nos animais gravemente acometidos que possam, eventualmente, sobreviver sem desenvolver a doença como sequela.

\section{REFERÊNCIAS}

Alves G.E.S., Ribeiro Filho J.D., Oliveira H.P. \& Abreu J.M.G. 2005. Tratamento da compactação experimental do cólon maior em eqüinos: resultados de laboratório e exames bioquímicos. Arq. Bras. Med. Vet. Zootec. 57(3):281-287.

Bailey S.R., Adair H.S., Reinemeyer C.R., Morgan S.J., Brooks A.C., Longhofer S.L. \& Elliott J. 2009. Plasma concentration of endotoxin and platelet activation in the developmental stage of oligofructoseinduced laminitis. Vet. Immunol. Immunopathol. 129(3-4):167-173.

Belknap J.K. \& Black S.J. 2005. Review of the pathophysiology of the developmental stages of equine laminitis. 51st Annual Convention of the American Association of Equine Practitioners (AAEP), Seattle, WA, USA. Disponível em: http://www.ivis.org/proceedings/aaep/2005/ belknap/chapter.asp?LA=1

Black S.J., Lunn D.P., Yin C., Hwang M., Lenz S.D. \& Belknap J.K. 2006. Leukocyte emigration in the early stages of laminitis. Vet. Immunol. Immunopathol. 109:161-166.

Campebell R.C., Peiro J.C., Rosa P.C.S., Valadão C.A.A. \& Bechara G.H. 2007. Endotoxemia por lipopolissacarídeo de Escherichia coli, em eqüinos: efeitos de antiinflamatórios nas concentrações sérica e peritoneal do fator de necrose tumoral alfa (TNF- $\alpha$ ). Arq. Bras. Med. Vet. Zootec. 59(4):837-843.

Dong H.P., Chen H.W., HSU C., Chiu H.Y., Lin L.C. \& Yang R.C. 2005. Previous heat shock treatment attenuates lipopolysaccharideinduced hyporesponsiveness of platelets in rats. Shock 24(3):239244.

Fernandes W.R., Coelho C.S., Marques M.S., Baccarin R.Y.A. \& Silva
L.C.L.C. 2003. Revisão de 26 casos clínicos de duodeno-jejunite proximal em eqüinos (1996-2000). Ciência Rural 33(1):97-102.

Galey F.D., Whiteley H.E., Goetz T.E., Kuenstler A.R., Davis C.A. \& Beasley V.R. 1991. Black walnut (Juglans nigra) toxicosis: A model for equine laminitis. J. Comp. Pathol. 104:313-326.

Garner H.E., Coffman J.R., Hahn A.W., Hutcheson D.P. \& Tumbleson M.E. 1975. Equine laminitis of alimentary origin: An experimental model. Am. J. Vet. Res. 36:441-444.

Hurley D.J., Parks R.J., Reber A.J., Donovan D.C., Okinaga T., Vandenplas M.L., Peroni J.F. \& Moore J.N. 2006. Dynamic changes in circulating leukocytes during the induction of equine laminitis with black walnut extract. Vet. Immunol. Immunopathol. 110(3-4):195-206

Jain N.C. 1986. Schalm's Veterinary Hematology. Lea and Febiger, Philadelphia, p.141.

Koury J.C.A., Lacerda H.R. \& Barros Neto A.J. 2006. Características da população com sepse em unidade de terapia intensiva de hospital terciário e privado da cidade do Recife. Revta Bras. Terap. Intensiva 18(1):52-58.

Loftus J.P., Belknap J.K. \& Black S.J. 2006. Matrix metalloproteinase-9 in laminae of black walnut extract treated horses correlates with neutrophil abundance. Vet. Immunol. Immunopathol. 113:267-276.

Loftus J.P., Black S.J., Pettigrew A., Abrahansen E.J. \& Belknap J.K. 2007a. Early laminar events involving endothelial activation in horses with black walnut-induced laminitis. Am. J.Vet. Res. 68(11):1205-1211.

Loftus J.P., Belknap J.K., Stankiewicz K.M. \& Black S.J. 2007b. Laminar xanthine oxidase, superoxide dismutase and catalase activities in the prodromal stage of black-walnut induced equine laminitis. Equine Vet. J. 39(1):48-53

Maitra S.R., Wojnar M.M. \& Lang C.H. 2000. Alterations in tissue glucose uptake during the hyperglycemic and hypoglycemic phases of sepsis. Shock 13(5):379-385.

Moore J.N.A. 2001. Perspective on endotoxemia. AAEP Proceedings 46:61-74.

Moore R.M., Bertone A.L., Muir W.W., Stromberg P.C. \& Beard W.L. 1994. Histologic evidence of reperfusion injury in the large colon of horses after low-flow ischemia. Am. J. Vet. Res. 55(10)1434-1443

Moore J.N., Garner H.E. \& Coffman J.R. 1981. Haematological changes during development of acute laminitis hypertension. Eq. Vet. J. 13:240242.

Nourian A.R., Baldwin G.I., Van Eps A.W. \& Pollitt C.C. 2007. Equine laminits: Ultrastructural lesions detected 24-30 hours after induction with oligofructose. Equine Vet. J. 39(4):360-364

Parsons C.S., Orsini J.A., Krafty R., Capewell L. \& Boston R. 2007. Risk factors for development of acute laminitis in horses during hospitalization: 73 cases (1997-2004). J. Am. Vet. Med. Assoc. 230(6):885-889.

Pollitt C.C. 1996. Basement membrane pathology: A feature of acute equine laminitis. Eq. Vet. J. 28:38-46.

Pollitt C.C. 1999. Equine laminitis: A revised pathophysiology. AAEP Proceedings 45:188-192.

Rhoads W.S., Barton M.H. \& Parks A.H. 1997. Small colon impactions in horses: 84 cases (1986-1996). AAEP Proceedings 43:248-249.

Rio Tinto J.J.M., Alves G.E.S., Faleiros R.R., Santos R.L., Marques Júnior A.P. \& Melo E.G. 2004. Utilização de hidrocortisona em eqüinos submetidos à isquemia e reperfusão no jejuno e suas conseqüências sob o cório lamelar. Arq. Bras. Med. Vet. Zootec. 56(3):292-299.

Silva M.A.M.L., Teixeira L.B.C., Geraldo Júnior C.A., Martin P.R., Silva R.D., Oshikoshi W. \& Doretto J.S. 2007. Coagulação intravascular disseminada (CID): revisão da literatura e relato de dois casos. Bolm Med. Vet. 3(3):3-16.

Zerpa H., Vega F., Vasquez J., Ascanio E., Campos G., Sogbe E., Romero E., Ascanio M. \& Garcia H. 2005. Effect of acute sublethal endotoxaemia on in vitro digital vascular reactivity in horses. J. Vet. Med. A, Physiol. Pathol. Clin. Med. 52(2):67-73. 